\title{
What Significance Will Digital Twin Have in an Industrial Environment in the Future?
}

\author{
Tony Jose \\ Department of Automation and Computer Science \\ Harz Univeristy of Applied Sciences \\ Wernigerode, Germany \\ u34958@hs-harz.de
}

\author{
Gittil Sagar \\ Department of Automation and Computer Science \\ Harz Univeristy of Applied Sciences \\ Wernigerode, Germany \\ u34984@hs-harz.de
}

\begin{abstract}
This paper is an attempt to forecast the potential application of Digital Twin in an industrial environment by adopting scenario analysis as part of technology monitoring. In recent years Digital Twin has become one of the most emerging topics in both the tech industry and academia. This field is the latest addition to industry 4.0. It has paved a great growth opportunity in the fields of Healthcare, manufacturing, and smart city areas. The Digital twin can be defined as mirroring the physical entities into a virtual environment where the status, health, and control of these entities can be monitored and controlled. It is a field where Artificial intelligence and the Internet of Things play a major role in the handshake between the two environments.

In this paper we have done a thorough analysis to find out the possible future outcome of this technology in the manufacturing industry from the perspective of our fictious company. These scenarios expose various challenges and opportunities our organization may face in our future endeavour.
\end{abstract}

Keywords Digital Twin(DT), Industry 4.0, DigiX, Internet of Things(IoT), Industrial IoT(IIoT), Artificial Intelligence, Scenario Analysis,Predictive Maintenance

\section{INTRODUCTION}

The latest Industrial revolution is termed Industry 4.0. It is a combination of automation and digitization of manufacturing using various physical and digital components with the help of data analytic and IoT. The main aim of Industry 4.0 is to enhance the performance of the industries by optimizing the various process and technologies [7]. A Digital Twin is seen as one of the main emerging enablers of Industry 4.0. A Digital Twin environment provides various applications like rapid system monitoring and real-time diagnostics through analytics [16]. We would be discussing in detail the significance of DT in an industrial environment in this paper.

\section{A. What is Digital Twin?}

- a) Nasa 2012 "A Digital Twin is an integrated multiphysics, multiscale, probabilistic simulation of an as-built vehicle or system that uses the best available physical models, sensor updates, fleet history, etc., to mirror the life of its corresponding flying twin" [15].

- b) Vrabic et al. 2018 "A Digital Twin is a digital representation of a physical item or assembly using integrated simulations and service data. The digital representation holds information from multiple sources across the product life cycle. This information is continuously updated and is visualized in a variety of ways to predict current and future conditions, in both design and operational environments, to enhance decision making" [15].

- c) Mandi 2019 "A Digital Twin is a virtual instance of a physical system (twin) that is continually updated with the latter's performance, maintenance, and health status data throughout the physical system's life cycle." [15]

From the above definitions we can identify that Digital Twin is a virtual model of a process, product, or service by realtime data collection using various sensors. This data can be locally decentralized or centrally stored in a cloud. This data is then evaluated to create a simulation known as Digital Twin.A Digital Twin comprises of three main elements [6].

- They are a physical item in real space.

- The Digital twin in software form.

- Data that links the physical entity and the software entity. Digital Twin are being more and more significant in various areas of industries.It helps the industries to enhance their overall performance by accurately predicting the outcomes thereby enhancing their business.The main potential application of Digital twin in industrial applications are production planning, product design, man-machine interaction,predictive maintenance, etc.

\section{B. DigiX}

DigiX is a fictitious company formed to provide service in the industrial area using the applications of Digital Twin.The main focus of DigiX is given below.

- Developing and analyzing the virtual industrial environment.

- Evaluating operational performance and cost reduction strategies for existing and new industrial environment.

We as an organization decided to select this technology as our field of business due the huge future potential this field can offer.It's application lies in various areas, but we have decided 
to focus on industrial application.This is because industry 4.0, which comprises of IIoT, AI, Machine learning, etc has already established a base for Digital twin [8].Therefore it would be a huge opportunity for us to get into this emerging field during an early stage.In this paper DigiX team has tried to create a clear picture of the future of Digital twin market using technology assessment.

\section{Methodology}

DigiX as an organization needs to foresee the potential of the Digital Twin industry to adopt various innovations in the organization and to tackle various challenges the risk that might arise in the future. So in this paper, we have done a technology assessment with the help of the scenario analysis method. We have selected scenario analysis for this technology monitoring. That is because the assessment of Digital Twin technology covers all five levels of innovation. They are competence, technology, functionality, performance, and market. The main objective of this analysis is to reduce the investment risk for the stakeholders and to achieve certain planning strategies. Scenario analysis helps the decisionmakers to deal with various uncertainties that may arise in the future.

\section{A. Scenario Analysis}

Scenario analysis provides a forward-looking view of operational risk that complements historical internal and external data. Such exercises allow those experts to better prepare to identify and manage the risk exposures through business decisions, risk mitigation efforts, and capital planning. Scenario analysis consists of five phases [12]. They are:

- Phase 1-Identification of scenario field

- Phase 2- Identification of Key factors

- Phase 3- Analysis of key factors

- Phase 4- Scenario Generation

- Phase 5- Scenario Transfer(Optional Phase)

\section{Detailed PROCEDURE OF SCENARIO ANALYSIS FOR} Digital TWIN

The scenario analysis for the significance of Digital Twin in an industrial environment is done in a systematic and formalized manner for DigiX. It has been carried out in four phases.

\section{A. Phase 1-Identification of The Scenario Field}

Identification of Scenario Field-This step involves identifying for what purpose the scenario has to be developed. It involves specifying the problem to be dealt with. It also involves other factors like "What must be integrated? How is the scenario field to be defined? [12]"

The identification of scenario field for Digital Twin was carried out based on the following findings and statistics.

- Digital Twin can be seen as a most promising technology trend in Gartner's recent report [10]

- Independent research Team Vendantix has predicted that the market for digital twin will grow from 787 million dollars in 2020 to 27.6 billion USD by 2040 [19].
- Around 89 percent of all IoT Platforms will contain some form of Digital Twinning capability by 2025 [1].

- Digital twinning will become standard feature/functionality for IoT Application enablement by 2027 [14].

- more than 92 percent of vendors recognize the need for IIoT APIs and the need for platform integration with digital twinning functionality for industrial verticals [14]

- Nearly 36 percent of executives across a broad spectrum of industry verticals understand the benefits of digital twinning and 53 percentage of them plan to incorporate within their operations by 2028 [14]

- Recently formed consortium named Digital Twin Consortium announced that its membership is growing rapidly including some major players like Autodesk, GE Digital etc [3].

From the above inference, we can say that the significance of digital twin is starting to take a shape in the industry 4.0 environment. Therefore we can conclude from the above findings that Digital twin technology is going to create a revolution across several industry sectors in the coming years.

\section{B. Phase 2- Identification of Key Factors}

Identification of Key factors is the primary goal in phase 2 of scenario analysis. The key factors can be those variables, parameters, trends, events, etc. These factors can have a positive/negative impact on the scenario field. Identification of these factors requires knowledge about the scenario field [12]. The Process of identifying factors within the scenario analysis process differs from case to case. It can be methods like survey, workshops, brainstorming, etc.

We have identified key factors which can affect the future development of the organization as well as technology. It was done using PESTAL analysis. A PESTLE analysis is a framework to analyze the key factors (Political, Economic, Sociological, Technological, Legal, and Environmental) influencing an organization from the outside [9]. It offers people professionals insight into the external factors impacting their organization. The analysis is flexible, so organizations can use it in a range of different scenarios. People professionals and senior managers can use the results to guide strategic decisionmaking [2].

For conducting the PESTAL we have done a thorough literature review and found out important factors using brainstorming. The below points are the result of the PESTAL analysis carried out for Digital Twin from the perspective of DigiX.

- Political - The effect of different laws and regulation, Encouraging new technologies,changing contractual frameworks, tax policies,labour laws etc.

- Economic- it consists of several factors like various investment policies, the effect of taxes on the economy, reduction of unemployment rates, and improvement of GDP. 


\begin{tabular}{|c|c|c|c|c|c|c|c|c|c|c|c|c|c|c|c|}
\cline { 2 - 13 } & $\mathbf{1}$ & $\mathbf{2}$ & $\mathbf{3}$ & $\mathbf{4}$ & $\mathbf{5}$ & $\mathbf{6}$ & $\mathbf{7}$ & $\mathbf{8}$ & $\mathbf{9}$ & $\mathbf{1 0}$ & $\mathbf{1 1}$ & $\mathbf{1 2}$ & $\mathbf{1 3}$ & $\mathbf{1 4}$ & $\begin{array}{c}\text { Active } \\
\text { Sum }\end{array}$ \\
\hline $\mathbf{1}$ & & 2 & 2 & 2 & 2 & 1 & 1 & 2 & 2 & 2 & 2 & 0 & 1 & 1 & 20 \\
\hline $\mathbf{2}$ & 2 & & 0 & 2 & 1 & 0 & 0 & 0 & 1 & 1 & 1 & 0 & 1 & 0 & 9 \\
\hline $\mathbf{3}$ & 2 & 1 & & 1 & 0 & 1 & 0 & 0 & 1 & 1 & 1 & 0 & 0 & 1 & 9 \\
\hline $\mathbf{4}$ & 2 & 1 & 0 & & 1 & 1 & 0 & 2 & 2 & 2 & 2 & 2 & 2 & 2 & 19 \\
\hline $\mathbf{5}$ & 1 & 1 & 1 & 2 & & 1 & 0 & 1 & 1 & 1 & 1 & 1 & 2 & 1 & 14 \\
\hline $\mathbf{6}$ & 0 & 2 & 1 & 2 & 1 & & 1 & 2 & 2 & 1 & 2 & 0 & 1 & 0 & 15 \\
\hline $\mathbf{7}$ & 0 & 0 & 1 & 1 & 1 & 2 & & 2 & 2 & 0 & 2 & 2 & 2 & 0 & 15 \\
\hline $\mathbf{8}$ & 0 & 0 & 1 & 0 & 1 & 1 & & & 1 & 2 & 0 & 0 & 0 & 2 & 8 \\
\hline $\mathbf{9}$ & 2 & 0 & 0 & 2 & 1 & 2 & 0 & & & 2 & 2 & 1 & 2 & 2 & 16 \\
\hline $\mathbf{1 0}$ & 2 & 0 & 0 & 2 & 0 & 2 & 0 & 2 & 2 & & 2 & 1 & 2 & 2 & 17 \\
\hline $\mathbf{1 1}$ & 2 & 0 & 0 & 2 & 1 & 2 & 0 & 0 & 2 & 2 & & 1 & 2 & 2 & 16 \\
\hline $\mathbf{1 2}$ & 2 & 1 & 0 & 2 & 0 & & 0 & 1 & 1 & 1 & 1 & & 2 & 1 & 12 \\
\hline $\mathbf{1 3}$ & 2 & 0 & 0 & 2 & 1 & 0 & 1 & 0 & 2 & 2 & 2 & 2 & & 2 & 16 \\
\hline $\mathbf{1 4}$ & 0 & 1 & 1 & 1 & 1 & 0 & 0 & 0 & 2 & 1 & 0 & 0 & 0 & & 7 \\
\hline Passive & 17 & 9 & 7 & 21 & 11 & 13 & 3 & 12 & 21 & 18 & 18 & 10 & 17 & 16 & \\
\hline sum & & & & & & & & & & & & &
\end{tabular}

Implementation of laws \& regulations Tax policy

Labour law

Encouraging new technologies GDP

Unemployment rates

Cultural aspects

Integrating production \& Simulation

Data security

Standardized design

Al

12 Effects on bio diversity

13 Resourcse consumption

14 Specific laws related to digitalization

: Identified Key Factors

Fig. 1. Cross Impact Analysis

\begin{tabular}{|c|c|c|c|c|}
\hline \multicolumn{5}{|c|}{ Characteristics of Key Factors } \\
\hline Key Factor & Description & Best & Base & Worse \\
\hline Laws and Regulation & $\begin{array}{l}\text { Specific Laws and Regulation related to } \\
\text { Digitilization }\end{array}$ & Favourable & Moderate & Unfavourable \\
\hline New Technologies & $\begin{array}{l}\text { Adoption of new technologies in the indus- } \\
\text { try }\end{array}$ & $\begin{array}{l}\text { Short time and } \\
\text { cheap }\end{array}$ & $\begin{array}{l}\text { moderate time } \\
\text { and cost }\end{array}$ & $\begin{array}{l}\text { long time and ex- } \\
\text { pensive }\end{array}$ \\
\hline Integrating Production and Simulation & $\begin{array}{l}\text { Lead to Successful implementation of Dig- } \\
\text { ital Twin }\end{array}$ & $\begin{array}{l}\text { Highly Success- } \\
\text { ful }\end{array}$ & $\begin{array}{l}\text { Moderately Suc- } \\
\text { cessful }\end{array}$ & Failure \\
\hline Data Security & $\begin{array}{l}\text { Protection of Digital Asset from unwanted } \\
\text { or unauthorized attacks }\end{array}$ & High & Medium & Less \\
\hline Standardized Design & $\begin{array}{l}\text { Adopting industry standard for DT architec- } \\
\text { ture }\end{array}$ & $\begin{array}{l}\text { Universal Accep- } \\
\text { tance }\end{array}$ & $\begin{array}{l}\text { Moderate Accep- } \\
\text { tance }\end{array}$ & No Acceptance \\
\hline Resource Consumption & $\begin{array}{l}\text { Consumption of energy and raw materials } \\
\text { for DT adoption }\end{array}$ & Less & Moderate & High \\
\hline Artificial Intelligence & $\begin{array}{l}\text { Involvement of AI in various DT applica- } \\
\text { tions }\end{array}$ & High & Medium & Low \\
\hline
\end{tabular}

Fig. 2. Characteristics of Key Factors

- Social- this is a combination of several aspects like a cultural barrier, career attitudes, and its influence on new work culture.

- Technological - Whenever a new technology comes into the industry several challenges comes along with it. Since Digital Twin is a highly complex technology integrating it with the existing production environment is a big challenge. Along with that maintaining a secured system by hardening the IT security is a major concern. Many of the challenges can be mitigated if they have an industry design standard. The Adoption of AI can also create a huge impact on this technology.

- Environmental- Various issues like the effect on biodiversity and resource consumption will be a major concern during the implementation of this technology. Also varying geographical locations might affect the development of this technology.

- Legal - Legal factor can be a combination of several legal frameworks related to digitalization and labor exploitation. 


\section{Phase 3-Analysis of Key Factors}

In the third phase of scenario analysis, the individual factors identified in phase two are subjected to analysis to formulate the future characteristics [12]. This analysis can be carried out using different methods. In this paper, we have conducted a cross-impact analysis to prioritize these factors based on the correlation between each factor [18]. The main factors are identified by considering the active and passive sum. Active sum shows how strongly the factor influence other and passive sum shows how much the factor is influenced by others.

In Cross Impact analysis the influence of each factor is a scale from -2 (Strongly inhibiting influence) to +2 (Promoting Influence). And the values in between show variation in influence.

After conducting cross-impact analysis on various factors we have identified the major factors that can have an impact on Digital Twin technology.Figure1 shows the result of the cross-impact analysis. In the figure, the highlighted factors can be seen as the major factors.

Later we have characterized each factor into three possible categories. They are Best Case, Base Case, and Worst Case.

The Table 2 shows the major characteristics of the selected key factors after cross-impact analysis. These salient characteristics described in this table becomes part of the future scenarios. In this table, we have listed the important key factors that can create an impact on the Digital Twin technology. Each case can produce three possible outcomes varying from Best to the worse case.

\section{Phase 4- Scenario Generation}

In this phase, various scenarios are generated based on analyzing the major key factors. The process of developing scenarios by merging the key factors can be done in different methods like narrative, literary procedure, or using mathematical techniques [12].Even if scenarios are theoretically possible the number of scenarios that can be made into practice is limited. However, in this paper, we have generated three practically possible scenarios that may arise for the future of Digital Twin technology.

By doing this we will be able to visualize the possible future outcomes. This will pave a path for corrections or drastic strategic innovations in the technology. This can help the management to portray a successful business plan to their investors.

The generated scenarios for the Digital Twin technology for DigiX are the following.

- Best Case Scenario-This scenario can be seen as the most successful scenario for Digital Twin. It consists of all the positive aspects which can encourage the universal adoption of Digital Twin in an industrial environment. In this case, it would start with the involvement of the government in encouraging industries to explore and utilize the advantages of Digital Twin. They would formulate favorable laws and regulations for the industries and the technology and make the adoption a smooth process. This will drive the industries to adopt Digital
Twin on a massive scale. It would socially be accepted by the masses and will integrate efficiently with existing employees. This will pave the way to new work culture. As per Industry this will be a game-changer for their production environment and would open a path for the smart industry. This would also help the industry to reduce unnecessary resource consumption based on smart raw material planning and digital prototype testing of the products. Global acceptance of this technology will be less complicated by creating a standardized design for DT architecture. This will encourage the industries even more to adopt this technology. Even though data security is a major concern in DT with the help of an efficient data security framework these hurdles can be reduced to a great extent. In this scenario, our organization will have a huge potential in the DT industry.

- Base Case Scenario- In this case, we can say that the Digital Twin technology would be adopted by the industry on a medium to large scale. Also, there will be an evolution of semi-digital industries where the Digital Twin technology would coexist with an existing industrial environment. We can also expect moderate support from the government and this might vary with respect to different countries. Some industries might recede from investing in Digital Twin technology due to various concerns. That can include various factors like data security concerns, high cost in implementing and maintenance of the Digital twin setup.

We can also expect major corporations to adopt these technologies and outrun the smaller ones. This case might also create an uneven distribution of power where huge organizations control the entire industry and forcing the small scale industries to suffer. This can also lead to the exploitation of labor.The patents and the intellectual properties will be owned by some companies which will prevent smaller industries to get into this field.

- Worst Case Scenario- There are multiple hurdles in this scenario that can lead to failure in adopting Digital Twin on a universal scale. It can be issues like unfavorable government policies and laws. Moral or ethical dilemma by the countries. Also, technical complexity like the integration of physical and virtual environment along with data security issues might force the industries to withdraw from investing in this field. Another major concern for industries can be a burden on company financial due to the high initial investment in setting up a Digital Twin environment.

Even though some organizations might try out Digital Twin technology majority would consider resisting the adoption of Digital Twin technology because of the above adversities. And this would force them to stay with the existing or improved system. This can lead to total failure of Digital Twin adoption and can lead to failure in the business model of DigiX. 


\section{Services OfFered by DigiX}

DigiX as a company is specialized in offering various services in the field of Digital Twin for industries. These services were designed based on the analysis of possible opportunities in this field. Below mentioned services are the main offerings of DigiX.

\section{A. Product development}

For any industry choosing the right machines for their production environment is a huge challenge. This might be due to various complexity in forecasting the production efficiency and reliability of the equipment's [11]. Therefore DigiX as an organization comes up with several digital prototyping, modeling, and analysis of these physical equipment. This can help the customers to set up an optimized industrial environment. This will also help in the reduction of raw materials needed for physical prototyping and testing. This will lead to a sustainable product development cycle.

\section{B. Design Customization}

Increasing the efficiency of machines of an existing factory is a tedious task for the organizations [17]. This is maybe due to a lack of precision in the design of the products or it can disturb the existing setup which might lead to more chaos. DigiX can help the organization to develop efficient designs with various permutations of the products. This can lead to personalized or customized products and services to their customers.

\section{Shop Floor Performance Improvement}

A new industry always has difficulty in setting up an optimized shop floor [21]. This is a bigger problem if it is an already finished shop floor. Therefore we can help the organization to design the shop floor from an early stage with the help of simulating it with a digital twin environment and help them find the most optimized shop floor design. We can also simulate multiple scenarios that can occur on the shop floor and find a suitable design for their shop floor. This can be applicable even for the improvement of the existing shop floor by monitoring and analyzing the shop floor performance.

\section{Predictive Maintenance}

It is the process of conducting maintenance to prevent predicted problems rather than conducting maintenance on fixed schedules or when an issue arises [13] Most organizations encourage predictive maintenance practices to prevent problems without incurring the cost of unnecessary or frequent maintenance. DigiX can provide a solution based on creating a digital twin of the equipment. We will be creating a virtual model which are driven based on real-time data. This data is continuously monitored and analyzed to predict the problems. This helps the organization to have a real-time status of the system health. We can also provide the solution for two areas in predictive maintenance. They are:-

- Virtual Physical twin: We will be developing the digital twin based on the knowledge of real-time data of the machines or equipment's [4]. This is practical only for organizations that want to enable predictive maintenance on a few systems.

- Virtual Statistical Twin: We can help the organization to develop a statistical model of machines using the historical sensor data without machine-specific knowledge [4]. This has got more advantage due to less involvement of the original equipment manufacturer.

\section{Discussion}

From the scenario analysis carried out for Digital Twin, we have noticed a significant change in the mindset of people. It is a mixed reaction where more people are positive towards digitalization of the industries. Also, the growth in the Digital Twin has a seen drastic increase recently. The Sudden influence of other fields like AI, IoT, and IIoT has also paved a way for Digital Twin [20]. The importance of a standard or universal architecture can enhance the adoption rate of this technology in industries. The lack of this standardization is one of the major challenges faced by the organization [5]. Therefore, a standard architecture can improve the opportunity for DigiX greatly.

From the analysis we were able to find out some of the major advantages and disadvantages of Digital Twin. One of the major advantages is increasing the reliability of the physical assets. Also, features like predictive maintenance can lead to less downtime and in return would result in better productivity and financial gain for the organization. It can also create a sustainable industrial environment by reducing resource consumption.

Some challenges that are presently causing concern for digital Twin are in Data Analytics and IoT aspects. The major issues in Data Analytics are problems related to Data, privacy, security, and infrastructure. In IoT, some of the challenges are common with Data Analytics. Apart from that, it has other issues like connectivity and standardized modeling.

The above-mentioned challenges are being addressed on priority in Digital Twin Technology. And we can expect a better and robust system that can overcome these challenges in the near future. Mentality of the people in adopting a new technology is a crucial factor in the success of any technology. Therefore we must make sure important industry stakeholders are involved from early stages of development. With this, we can expect industries to start exploring small prototyping of Digital Twin system in the upcoming future. Currently, Digital Twin is being tried out on a small scale. It is gradually moving towards bigger organizations. We can also expect more government support in the adoption of Digital Twin in the future by providing favorable laws and policies. 


\section{CONCLUSION}

This paper can be seen as a consolidated analysis carried out in various phases of scenario analysis. It can be used to figure out the potential of Digital Twin technology in the industrial environment. In our analysis we found out the major factors that can have an effect on the Digital Twin technology in the future.They are starting from standardized design, adoption of new technologies, creating a secure environment for DT system, and support of the government in various stages of the adoption of Digital Twin. .

From the result of the overall scenario analysis, we found that there three possible scenarios. All these scenarios have some amount of Digital Twin significance. In the Best case scenario, our organization can achieve significant opportunity and growth in the Digital Twin market. In the Base case scenario, we would have a moderate market and might have gradual growth in the future. In the worst-case scenario, we would still have some opportunities but growth would be limited.

Considering the opportunities and the potential for future growth in the Digital Twin field, DigiX as an organization has planned to focus on four major areas of Digital Twin. They are product development, design customization, shop floor performance improvement, and predictive maintenance. Therefore we would be able to diversify our service portfolio and lowering the risk of our investors.

\section{REFERENCES}

[1] https : / / www . prnewswire . com / news - release / the future- of-the-digital-twins-industry-to-2025/. [Online; accessed 07-02-2021].

[2] https : / / www . cipd . co . uk / knowledge / strategy / organisational - development/pestle - analysis - factsheet. [Online; accessed 04-02-2021].

[3] Digital Twin Consortium. digitaltwinconsortium.org/. [Online; accessed 05-02-2021].

[4] Cem Dilmegani. https : // www . research . aimultiple . com/predictive-maintenance/. [Online; accessed 08-022021]. 2021.

[5] Tolga Erol and Arif Furkan Mendi. "Digital Transformation revolution with Digital Twin technology". In: (2020).

[6] David Essex. https://www. searcherp.techtarget.com/ definition/digital-twin. [Online; accessed 06-02-2021]. 2020.

[7] Aidan Fullerand Zhong Fan. "Digital Twin:Enabling Technologies,Challenges and Open Research". In: (2020), pp. 1-21.

[8] He Zhang Fei Tao. "Digital Twin in Industry:State of the Art". In: (2019), Volume 15.

[9] Rishabh Ghotge and Elisabeth Vandeventer. "A global Analysis on Microgrids through the PESTAL Framework". In: (2020).

[10] Eyre Jonathan and Christopher Freeman. "Immersive Application of Industrial Digital Twin”. In: (2018).
[11] Atakan Kantarci. https : // www . research . aimultiple . com/digital - twin - application/. [Online; accessed 0602-2021]. 2021.

[12] Hannah Kosow and Robert Gassner. "Methods of Future and Scenario Analysis". In: (2008), pp. 1-133.

[13] Valentina Di Pasquale and Tsega Y Melesse. "Digital Twin Models in Industrial Operations:A systematic Literature Review". In: (2020), volume42, 267-272.

[14] Adil Rasheed and Trond Kvamsdal. "Digital Twin:Values, Challenges and Enablers From a Modelling Perspective". In: (2020), pp. 21980-22012.

[15] Roland Rose and George Von Wichwert. "About the importance of autonomy and Digtal Twin for the Future of Manufacturing". In: (2015), pp. 1-2.

[16] Rohan Basu Roy and Debashis Mishra. "Digital Twin:Current Scenario and Case stude on a Manufacturing process". In: (2020).

[17] Benjamin Schleich and Nabil Anwer. "Shaping The digital Twin for Design and Production Engineering". In: (2017), pp. 141-144.

[18] Serdar Seyda and Umut Asan. "Quantitative crossimpact analysis with time consideration". In: (2007).

[19] Twinview. https://www.twinview.com/insights/digitaltwin-spending/. [Online; accessed 07-02-2021].

[20] Priyan William Davis and Malarvizi Kumar. "A Digital Twin Framework for industry 4.0 enabling next-gen manufacturing". In: (2020).

[21] Cunbo Zhuang and Tian Miao. "The Connotation of Digital Twin, and the construction and application method of shop floor digital twin". In: (), volume68. 